\title{
Condition diagnosis of a practical bridge by monitor data of concrete cracks
}

\author{
H.X. LI \\ Department of Medical Imaging, Shandong Medical College, Jinan, China
}

B. JIN

Jinan Urban Construction Group, Jinan, China

Y. LIU

School of transportation science and Engineering, Harbin Institute of Technology, Harbin, China

\begin{abstract}
Concrete crack is a popular damage of practical concrete bridges, so it is deserve to investigate the changing condition of concrete cracks of practical bridges. Based on this point, in this study, structural health monitoring (SHM) technique was utilized for monitoring the variation of crack width of a practical bridge. Some de-tails of the SHM system were shown and analyzed in order to obtain the change of crack width indirectly. Structural strain crossed the crack of bridge beam were measured timely by using SHM technique, which could evaluate the variation of crack width of bridge. By this way, the condition of this bridge was assessed from the variation trend of concrete cracks.
\end{abstract}

KEYWORD: Bridges; Structural Health Monitoring; Structural strain; Concrete Cracks

\section{INTRODUCTION}

$\mathrm{SHM}$ is an effective technique for diagnosing the structural damage and assessing the condition of bridges ( $\mathrm{Li} \& \mathrm{Ou} 2008$ ). It is a combination of couples of technique like sensor technique, data mining methods, theory of damage diagnosis etc. (Ogilvie et al. 1998). With the development in past decades, it is gradually carried out for evaluating the structural condition of bridges (Sandhu et al. 2001).

Prestressed concrete bridge takes a huge ratio of the total numbers of bridges in China. For this type of bridge, some damages like the concrete cracks are very common (Lazarevic et al. 2003). In theory, the cracks should not be found for the full prestressed concrete bridges, but they cannot be avoided for the practical bridges due to the construction technique, environmental effect and design issues etc. (Aleksandar et al. 2004). Once the occurring of crack, the concrete cracks always develop gradually, which appears that the width of concrete crack widen with the operation of practical bridges (Sohn et al. 1999). Therefore, it is important to obtain the change process of the width of concrete cracks, which is the main point of this study.

At the end, the sketch of this study is organized as follows. Detailed information of the SHM design of a practical bridge were introduced in next section. The analytical results were analyzed and discussed in Section 3. At last, conclusions were drawn.

\section{CONCRETE CRACK MONITORING OF A PRACTICAL BRIDGE}

\subsection{Brief description of a practical bridge}

A practical bridge that is located at Guangshen highway in China is taken as an example in this study. The beam of this bridge is prestressed concrete hollow slab, and the structural section of this bridge is drawn in Fig. 1.

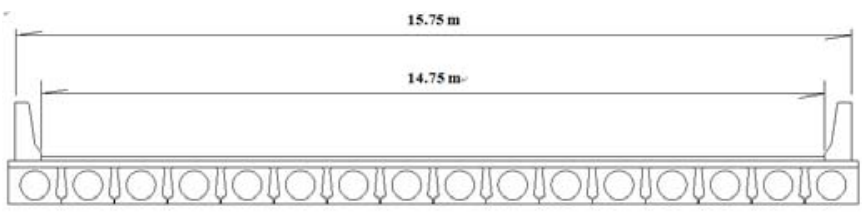

Figure 1. Section of prestressed concrete hollow slab

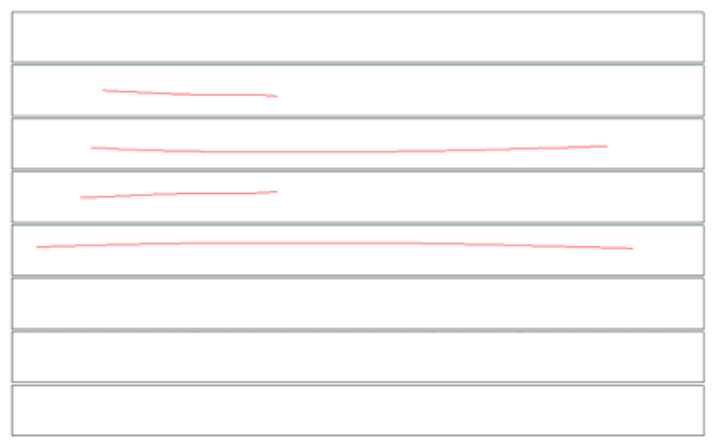

(a) Left span 


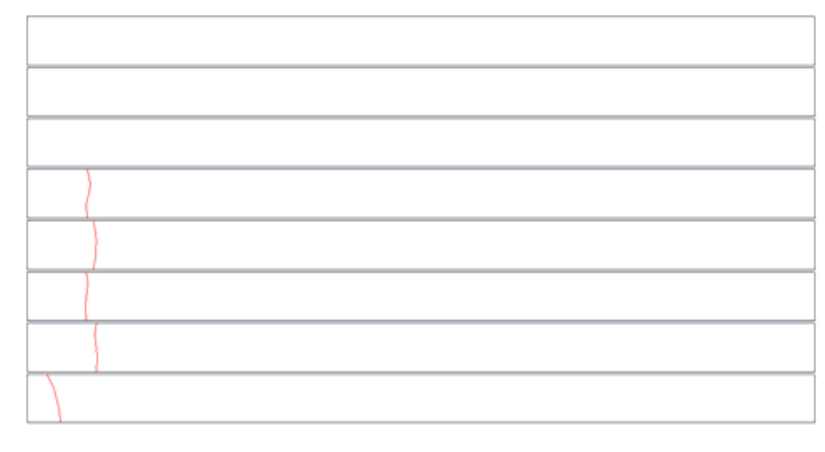

(b) Right span

Figure 2. Description of concrete cracks of a bridge

During the operation of last two decades, concrete cracks became the main damage mode of some bridges. Among the main types of concrete cracks, we found out that concrete cracks generated along the width direction of bridges taken a huge ratio. Otherwise, another main part of concrete cracks go to the ones occurred along the longitude direction of this bridge (Fig. 2). Furthermore, some cracks were found out that the width of cracks were bigger than the threshold value which was determined from Chinese bridge design code.

From the point of view of bridge safety, it is important to monitoring the long-term changing process of concrete cracks. Based on this point, a simple SHM system was designed and implemented by the management of this bridge. The width of concrete cracks were monitored by using FBG (Fiber Bragg Grating) sensors, and the detailed description are shown as follows.

\subsection{SHM system of concrete cracks}

For measuring structural strain, some types of sensors could be utilized. In this study, FBG sensor was selected as the main way for strain measurements because of its long-term durability.

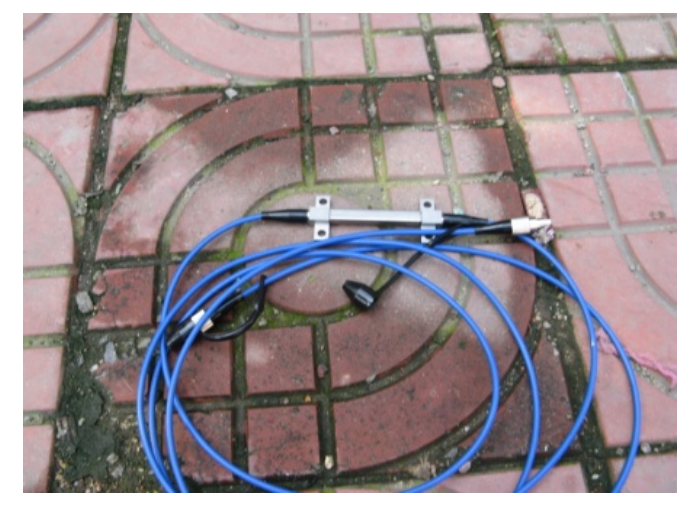

Figure 3. FBG sensor for measuring structural strain

Different characteristics would be shown in the type of sensors. Compared with FBG sensor, the former two kinds of sensors are relatively cheaper, being utilized widely for the structural strain meas- urement. However, monitoring the long-term variability of structural strain would be limited by the relative short-term durability of sensor. While longterm durability merit has lied in FBG sensors, so the long-term variation of structural strain is apt for this type of sensor if embedded into the structural interior. Therefore, FBG would be proper for obtaining the variability of concrete cracks of a practical bridge.

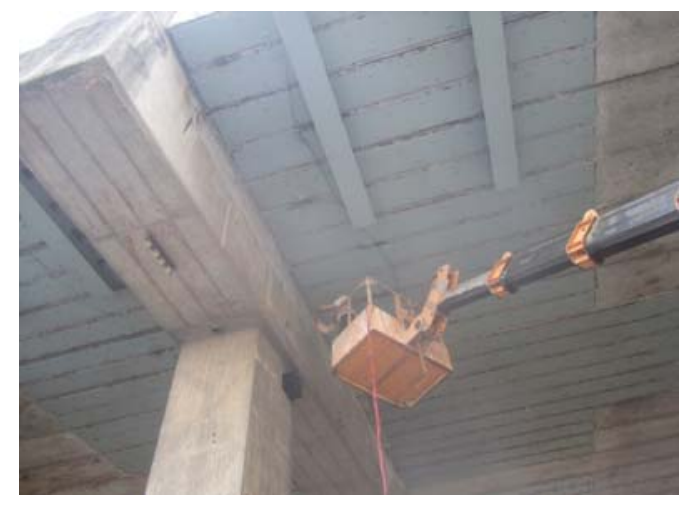

Figure 4. Set up of FBG sensor

For this bridge, 22 concrete cracks were selected for monitoring, including 14 longitudinal cracks and 8 lateral cracks of bridge. One strain sensor was set crossed one normal crack except that two extremely long cracks were placed for two strain sensors respectively. The placement of sensors were shown in Figure 4 and Figure 5. And the data acquisition system of FBG sensor was shown in Figure 6.

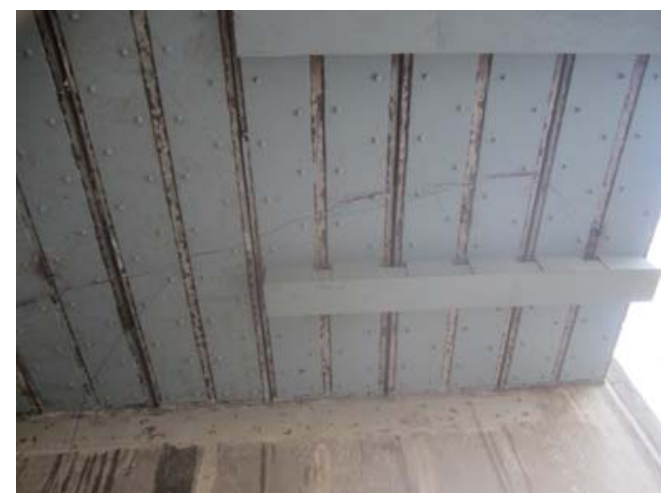

Figure 5. Protection cover of FBG sensor

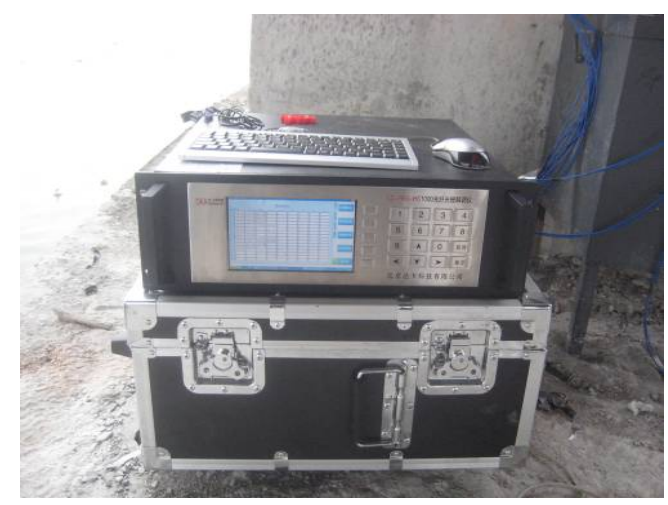

Figure 6. Data acquisition system of FBG sensor 


\section{ANALYTICAL RESULTS OF MONITORING DATA}

The width variation of each concrete crack was obtained by the direct analysis of the monitored strain. Usually, the variation of the wave length of the sensors caused by the coupled actions of temperature effects and exterior loads could be calculated as follows:

$\mathrm{d} \lambda=\alpha_{\varepsilon}\left(\varepsilon_{0}+\alpha_{\mathrm{c}} \mathrm{d} T\right)+\alpha_{\mathrm{T}} \mathrm{d} T$

Furthermore, Equation (1) could be transformed as follows:

$$
\begin{aligned}
& \varepsilon_{0}=\frac{1}{\alpha_{\varepsilon}} \mathrm{d} \lambda-\left(\frac{\alpha_{\mathrm{T}}}{\alpha_{\varepsilon}}+\alpha_{\mathrm{c}}\right) \mathrm{d} T \\
& \varepsilon_{0}=\alpha^{\prime}\left(\lambda-\lambda_{0}\right)-\alpha_{\mathrm{T}}^{\prime}\left(\lambda_{\mathrm{T}}-\lambda_{\mathrm{T} 0}\right)
\end{aligned}
$$

where $\varepsilon_{0}=$ structural strain under exterior loads; $\alpha^{\prime}=$ strain coefficient of each sensor; $\lambda=$ measured wave length of each sensor; $\lambda_{0}=$ initial wave length of each sensor (determined by the first measurement); $\alpha_{\mathrm{T}}^{\prime}=$ temperature coefficient of each testing point (including the effects on the structure and sensors); $\lambda_{\mathrm{T}}=$ measured wave length of the temperature sensor; $\lambda_{\mathrm{T} 0}=$ initial wave length of the temperature sensor (corresponding to the same state of $\left.\lambda_{0}\right)$; and $\alpha_{T}=$ temperature coefficient of each sensor.

To evaluate the variability of concrete crack, the reference point is deemed according to the initial strain, and the relative crack width variation is calculated by the difference between monitored strain and the reference value. Based on this theory, the crack width variation obtained from Oct. 2014 to Mar. 2015 were shown from Figure 7 to Figure 12.

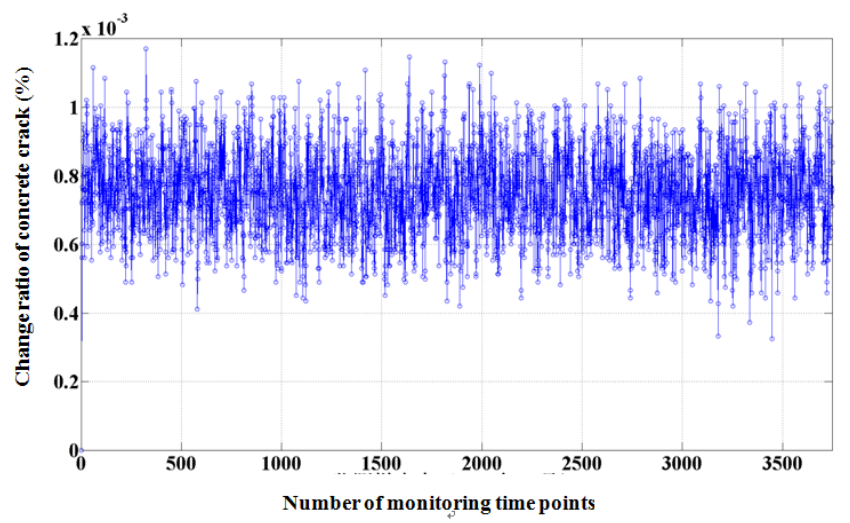

Figure 7. Change ratio of width of concrete crack (Oct. 2014)

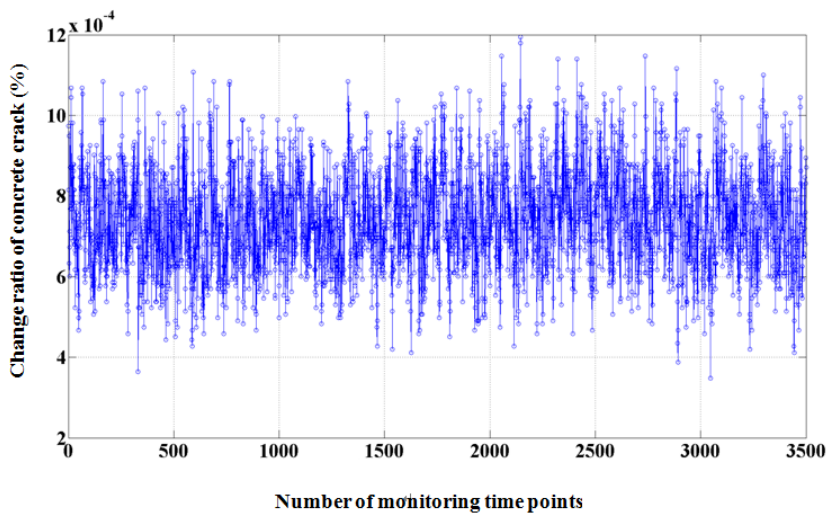

Figure 8. Change ratio of width of concrete crack (Nov. 2014)

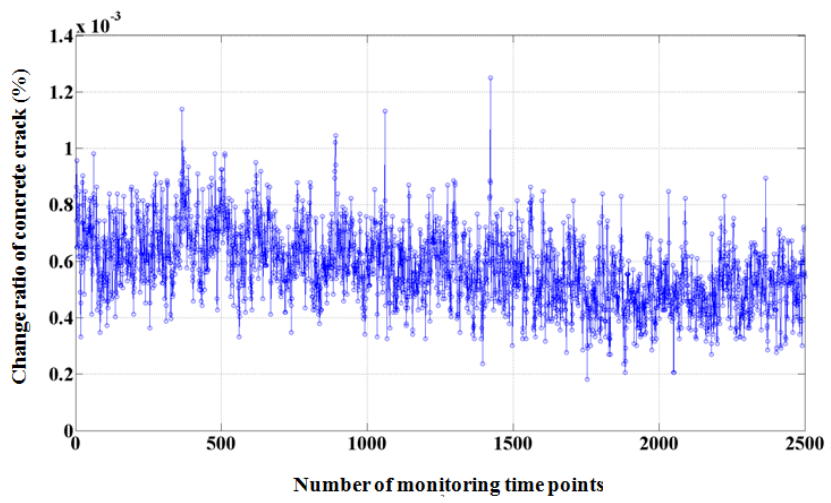

Figure 9. Change ratio of width of concrete crack (Dec. 2014)

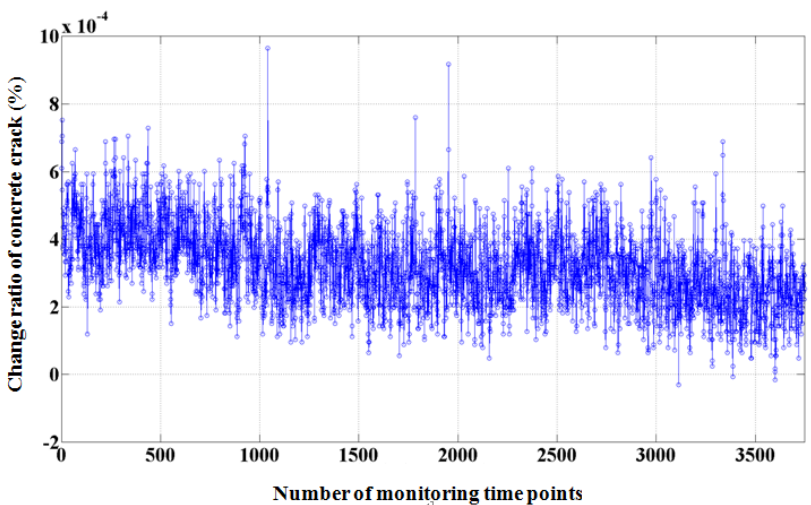

Figure 10. Change ratio of width of concrete crack (Jan. 2015)

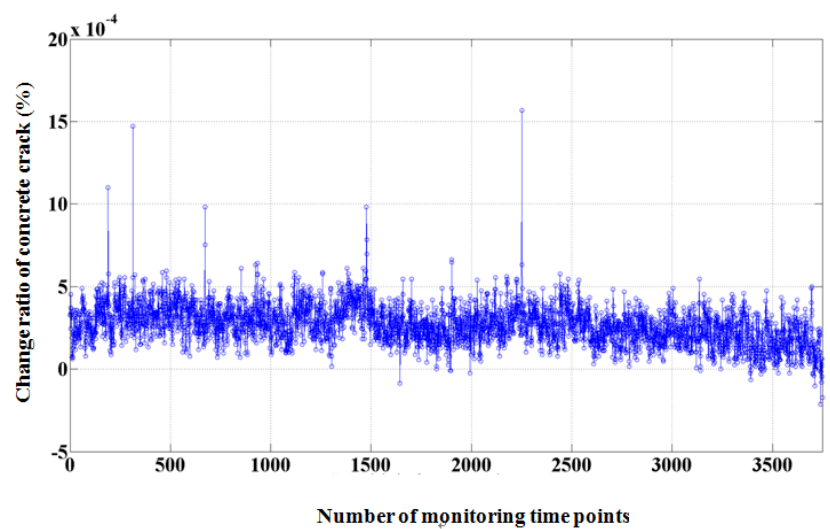

Figure 11. Change ratio of width of concrete crack (Feb. 2015) 


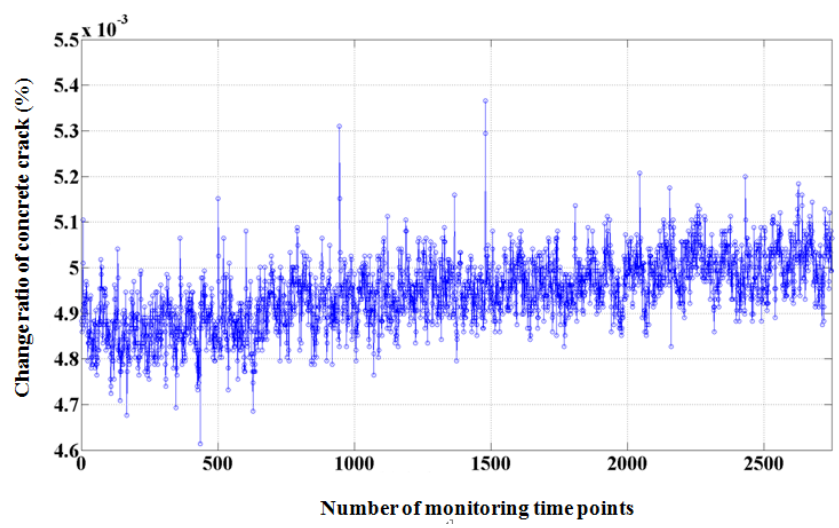

Figure 12. Change ratio of width of concrete crack (Mar. 2015)

\section{CONCLUSIONS}

In this paper, a practical application of SHM technique to investigate the variability of concrete cracks of bridges was carried out. Firstly, the introduction was about the detailed monitoring system information of a practical bridge. Secondly, the width variation of concrete cracks was obtained continuously by FBG sensors. Finally, the sensor monitoring data were analyzed, and the results showed no abnormal variation lied in the crack width for the half of year' monitoring. From this point of view, the bridge condition is safe and stable.

\section{REFERENCES}

Aleksandar, L. et al. 2004. Effective localized regression for damage detection in large complex mechanical structures. The 10th ACM SIGKDD International Conference on Knowledge Discovery and Data Mining: 22-25.

Lazarevic, A. et al. 2003. Localized prediction of multiple target variables using hierarchical clustering. Proceedings of the IEEE International Conference on Data Mining: 58-62.

Li, H. \& Ou, J.P. 2008. Structural health monitoring in mainland of china: theories, technologies and applications. Proceedings of the 2nd Asia-Pacific Workshop on Structural Health Monitoring: 14-22.

Ogilvie, T. et al. 1998. Use of data mining techniques in the performance monitoring and optimization of a thermal power plant. Knowledge Discovery and Data Mining, IEEE Colloquium: 7.

Sandhu, S.S. et al. 2001. Damage prediction and estimation in structural mechanics based on data mining. The 7th ACM SIGKDD International Conference on Knowledge Discovery and Data Mining/4th Workshop on Mining Scientific Datasets: 26-29.

Sohn, H. et al. 1999. An experimental study of temperature effect on modal parameters of the Alamosa Canyon Bridge. Earthquake Engineering and Structural Dynamics 28(8): 79-97. 